\section{Gategory}

Synthesis of

Heterocycles

\section{Key words}

pyrazoles

iron catalysis

hydrazones
N. PANDA,* A. K. JENA (NATIONAL institute OF TECHNOLOGY, ROURKELA, INDiA)

Fe-Catalyzed One-Pot Synthesis of 1,3-Di- and 1,3,5-Trisubstituted Pyrazoles from Hydrazones and Vicinal Diols

J. Org. Chem. 2012, 77, 9401-9406.

\title{
Iron-Catalyzed Synthesis of Pyrazoles from Hydrazones
}

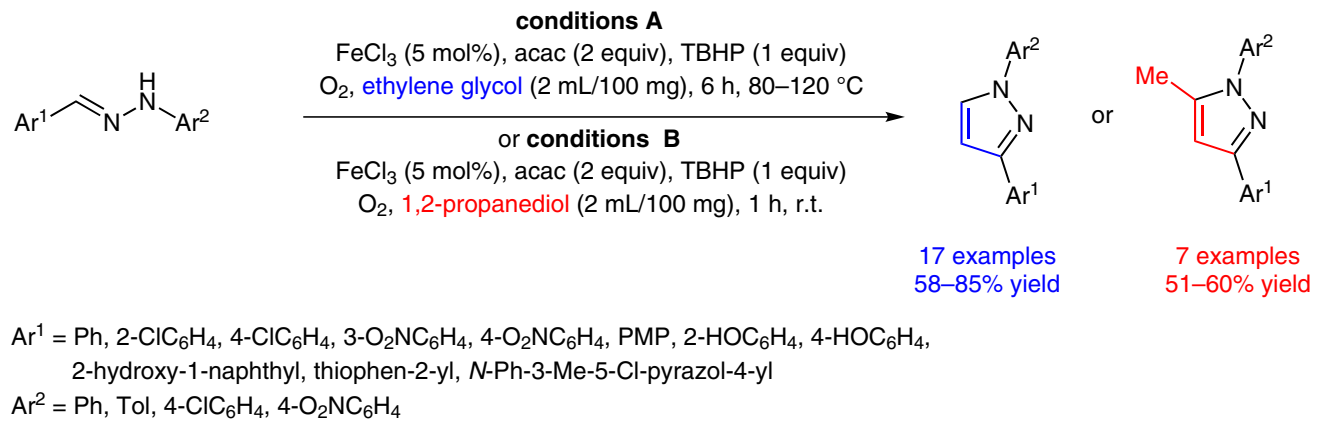

17 examples $58-85 \%$ yield

or

7 examples $51-60 \%$ yield

$\mathrm{Ar}^{1}=\mathrm{Ph}, 2-\mathrm{ClC}_{6} \mathrm{H}_{4}, 4-\mathrm{ClC}_{6} \mathrm{H}_{4}, 3-\mathrm{O}_{2} \mathrm{NC}_{6} \mathrm{H}_{4}, 4-\mathrm{O}_{2} \mathrm{NC}_{6} \mathrm{H}_{4}, \mathrm{PMP}, 2-\mathrm{HOC}_{6} \mathrm{H}_{4}, 4-\mathrm{HOC}_{6} \mathrm{H}_{4}$,

2-hydroxy-1-naphthyl, thiophen-2-yl, $\mathrm{N}$-Ph-3-Me-5-Cl-pyrazol-4-yl

$\mathrm{Ar}^{2}=\mathrm{Ph}, \mathrm{Tol}, 4-\mathrm{ClC}_{6} \mathrm{H}_{4}, 4-\mathrm{O}_{2} \mathrm{NC}_{6} \mathrm{H}_{4}$

Significance: The synthesis of pyrazoles from a condensation of $\alpha$-hydroxycarbonyl compounds with aryl hydrazones is reported. The requisite intermediate $\alpha$-hydroxy carbonyl compounds are formed by an iron-based in situ oxidation of vicinal diols by tert-butyl hydroperoxide (TBHP). Whilst studying optimization, it was found that by adding a ligand, the desired pyrazoles are obtained directly. Acetylacetone (acac) is the optimal ligand with anhydrous $\mathrm{FeCl}_{3}$ being the optimal iron source. The reaction is lower yielding under nitrogen or air, as opposed to an oxygen balloon. The substrate scope was reasonably well studied, although incorporation of vicinal diols beyond the presented two would have been welcome. A limitation seems to be the electron-withdrawing substituents on nitrogen, as the reaction with $\mathrm{Ar}^{2}=4-\mathrm{O}_{2} \mathrm{NC}_{6} \mathrm{H}_{4}$ fails.
Comment: The presence of a pyrazole motif in several drugs and pesticides has undoubtedly stimulated the development of new and complementary methods for their synthesis. In particular, transition-metal catalysis has been successfully used for the synthesis of polysubstituted pyrazoles. The reported method draws upon the pioneering work of Bolm, Beller and Repo in the field of ironcatalyzed oxidation (e.g., C. Bolm, M. Nakanishi Adv. Synth. Catal. 2007, 349, 861). The protocol utilizes inexpensive materials, and the conditions are mild especially for the synthesis of 1,3,5-trisubstituted pyrazoles (room temperature) which should have warranted further studies on substituted diols. The drawback is the necessity for super-stoichiometric amounts (2 equiv) of a ligand, which is quite wasteful. 